\title{
sciendo
}

\author{
BULGARIAN ACADEMY OF SCIENCES
}

CYBERNETICS AND INFORMATION TECHNOLOGIES • Volume 21, No 2

Sofia $\bullet 2021$

Print ISSN: 1311-9702; Online ISSN: 1314-4081

DOI: $10.2478 /$ cait-2021-0026

\section{A Centralized Model Enabling Channel Reuse for Spectrum Allocation in Cognitive Radio Networks}

\author{
Monisha Devi, Nityananda Sarma, Sanjib K. Deka \\ Department of Computer Science \& Engineering, Tezpur University, 784028 Assam, India \\ E-mails:mnshdevi@gmail.com nitya@tezu.ernet.in sdeka@tezu.ernet.in
}

\begin{abstract}
Cognitive Radio $(C R)$ is an advanced technology, which intends to boost the radio spectrum utilization. On perceiving the spectrum holes, next there is a need to provide a fair distribution of the vacant licensed channels amongst Secondary Users (SUs) during the spectrum allocation process. In this context, our paper introduces two allocation models to resolve the spectrum allocation problem. Initially, we design a simple centralized model to assign the channels. Then, we extend it to a centralized fair allocation model that aims to impart a better utilization of the free channels. Both approaches assign a common channel to a group of noninterfering SUs simultaneously. This facilitates spectrum reuse. The constraint related to dynamics in spectrum opportunities in CR is handled during channel allocation. Simulation study analyzes the proposed approaches with an existing allocation mechanism and reveals the performance improvement of centralized fair allocation model in terms of spectrum utilization.
\end{abstract}

Keywords: Cognitive radio, dynamic spectrum access, spectrum allocation, spectrum opportunities, channel reuse.

\section{Introduction}

Widespread development of wireless technologies across the world leads to inadequacy of the radio spectrum. On the contrary, conventional fixed spectrum assignment policy of government bodies imposes rights regarding spectrum access upon the license owner. But, according to reports presented by an authorized organization called Federal Communications Commission (FCC) [1], it has been observed that a significant portion of the licensed spectrum remains largely unused by their legitimate owners (either licensed users or primary users) at any given time or location. This instigates the generation of spectrum holes. Therefore, the uneven utilization of the radio frequencies results in an ineffectual use of the spectrum bands. Under such consideration, a new communication paradigm called Cognitive Radio (CR) [2-4] has been introduced as a solution to the spectrum scarcity problem. Operating upon the concept of Dynamic Spectrum Access (DSA) [5], CR enables the unlicensed users (or secondary users) to exploit dynamically the Spectrum 
OPportunities (SOPs) in such a way that there is no any disruptive interference to Primary Users (PUs). With its opportunistic behaviour, CR incorporates different network functionalities, which allow the Secondary Users (SUs) to take advantage of the spectrum holes. On realizing the free channels during the spectrum sensing phase, next there arises a need to share efficiently these channels amongst the SUs. This necessitates the development of allocation models, which takes the responsibility of providing an orderly distribution of the channels. So, radio spectrum allocation encourages the development of allocation mechanisms by formulating different design models and taking into consideration some prerequisite requirements such that spectrum utility gets enhanced along with improved network performance. Overall, the spectrum sharing process in Cognitive Radio Networks (CRNs) aims to make the utmost use of the idle licensed channels amongst the SUs.

Several spectrum alocation models have been already designed to facilitate channel allocation in CRN [6]. However, the existing models in literature miss out an important constraint due to which the network throughput can get affected. In CRN, all channels that are free may not be available to every SU. This happens due to different hardware constraints that can arise during the spectrum sensing process resulting in different SU capabilities [7]. So, during the allocation, if an SU is assigned a channel which is actually unavailable at the SU, then the SU cannot proceed with its transmission which in turn degrades the network throughput. Under such circumstance, taking an assuming that all channels are available to every SU does not showcase a practical scenario in CRN. Hence, it is preferable to study the channel availability of every SU before starting the allocation process.

Motivated with these observations, this paper proposes a spectrum allocation mechanism for CRN, which takes a central entity as the decision-making body for different allocation strategies. We extend the work carried out in [8] where singlewinner allocation has been followed for the available channels. However, by incorporating channel reuse in this model, we can allow multiple non-interfering SUs to get hold of a common channel. This further helps to improve the spectrum utilization, since more number of SUs can use the free channels. Also, one SU can request for more than one channel. With such an allocation constraint, an SU can continue its transmission without any disturbance even when a PU returns back to its owned channel. As such, the proposed mechanism tries to allocate the vacant channels such that one channel can be allotted to more than one user at a time, and also one SU can exploit more than one channel at a time. Moreover, to decide the channel allocation pattern, dynamics in Spectrum OPportunities (SOPs) has been considered which studies the channel availability of every SU before performing the allocation. Initially, a simple centralized model tries to allocate the vacant channels amongst SUs. But, to make the utmost use of the available channels, we design another centralized fair allocation model which further enhances the overall spectrum usage. Simulation studies are carried out to evaluate the performance of the proposed mechanism. Both approaches are compared with an existing allocation mechanism in terms of spectrum utilization, user satisfaction, spectrum reuse and throughput where centralized fair allocation scheme outperforms in its performance. 
The rest of the paper is organized as follows. In Section 2, we present the literature review. The proposed mechanism is described in Section 3, which contains an illustration of the system model and the proposed mechanism. Section 4 discusses the performance evaluation of the model and finally we conclude the paper in Section 5 .

\section{Related work}

Cognitive radio provides a platform to ameliorate the spectrum utilization efficiency. Spectrum sharing plays a key role in CR, which renders a fair distribution of the spectrum holes. Authors in [9] discuss several allocation models that have been designed with different network requirements to facilitate the use of radio spectrum. In [10], an oligopoly market is designed for trading the channels where a noncooperative game formulation defines the Nash equilibrium as a solution for maximizing profit of SUs. A graph coloring based channel assignment is carried out in [11], where by applying different colors to every channel the authors carry out the allocation process but in such a way that no two adjacent vertices (SUs) are given the same colored channel. Another channel allocation approach relates to a bipartite graph design in [12] where finding a maximal weight matching in the graph accounts to a fair allocation. Two vertex sets correspond to the set of channels and the set of SUs respectively where single-channel allocation is being performed with an intension to maximize the spectrum utilization. Deploying of genetic algorithm provides a different perspective for spectrum assignment. Such an approach is discussed in [13] where a conflict-free channel assignment is represented as a chromosome string with different gene patterns. In [14], a randomized rounding algorithm has been formulated which operates for heterogeneous channel allocation. Another design model for power allocation is studied in [15], which allows the SUs to transmit along with the PUs while maintaining the interference below a threshold level. [16] uses the concept of multiple knapsack problem design where discontinues spectrum holes are aggregated to form widely available spectrum knapsack and two spectrum allocation algorithms, optimal and suboptimal, assign the knapsack while maximizing spectrum utilization. Further, due to the perceived fairness in resource allocation, auction can be considered as another design model. In [17], authors formulate a bandwidth auction where a dynamic updating algorithm plans the allocation strategy for both single-PU and multi-PU scenarios. A strategy-proof auction mechanism is discussed in [18], which performs allocation amongst shared and exclusive-use devices by following bucketing and ironing techniques such that it achieves a monotone allocation rule. Again, [19] designs two truthful online auction models with unknown number of bidders competing for randomly available spectrum bands. First price auction model has been applied in [20], which allocates the auctioned channel to the highest bid user and intends to obtain a higher market profit. Subsequently, a double-auction model that aims at optimizing the profit and energy of CRN is developed in [21], which uses SINR model to carry out single-channel allocation. Another double-auction model called PreDA [22] makes its channel request based on a preference list that is generated based on SINR values of the 
channels and accordingly the model assigns channels to the most preferable users. The allocation model in [23] studies both seller side and buyer side auctions in a dissembled manner where seller side auctions its resources following a standard auction, such as Vickrey Clarke Groves (VCG), whereas buyer side allows partitioning of conflict graphs to determine winners along with their price. A merge process combines the winner sets from subgraphs to provide conflict-free spectrum distribution. To enable simultaneous sharing of the channels between PUs and SUs, an auction mechanism has been designed in [24], which works using the concept of interference temperature to prevent the PUs from any harmful disturbance. Hence, the state-of-art on spectrum allocation approaches for CRN unveils that the problems arising due to dynamics in SOPs in a CR environment has not been tackled in the existing approaches. This paper plans to incorporate this constraint while proposing the allocation model for CRN.

\section{Spectrum allocation mechanism for CRN}

\subsection{System model}

In the proposed model, we consider a cognitive radio network, consisting of SUs, which coexist with a primary network, having PUs. $M$ number of free channels are left unused by the PUs, which can be dynamically accessed, by $N$ number of SUs. The set of channels is given as $\Delta=\{1,2,3, \ldots, M\}$ and the set of SUs is given as $\Omega=\{1,2,3, \ldots, N\}$. We assume that $N>M$. The primary responsibility of channel allocation is taken up by a central entity, $C_{\mathrm{E}}$, who makes the decision based on the preference shown by the SUs for their desired channels. All SUs are considered to be within the transmission range of $C_{\mathrm{E}}$. Initially, before starting the allocation, every SU senses for its available channels, which may differ due to different SU capabilities. This constructs a channel vector $\Lambda_{i}=\left\{i_{1}, i_{2}, \ldots, i_{j}, \ldots, i_{N_{i}}\right\}$ at each SU $i$ which holds the free channels for the SU. Here, $i_{j}$ represents the $j$-th channel of SU $i$ and $N_{i}=\left|\Lambda_{i}\right|$ gives the number of channels available at SU $i$. To manage the channel availability of each $\mathrm{SU}$ at $C_{\mathrm{E}}$, it keeps a channel availability matrix. During the sharing process, every channel is picked up one-by-one by the $C_{\mathrm{E}}$ in a sequential order. Also, in this model, we have tried to exploit the reusability feature of radio spectrum. While taking into concern the interference constraint, SUs that are geographically far apart can use the same frequency band simultaneously which results in enhanced spectrum utilization. But, SUs in close proximity are subjected to interference which abstains them from using the same channel. So, to show the interfering SUs, an interference matrix has been maintained. Allocating an SU with more than one channel can be advantageous because, for an SU while transmission, if the channel needs to be released due to PU activity, the SU can shift to another available channel for seamless communication. Such an act avoids any interruption in the transmission process of the SU. As such, we confine the proposed mechanism to the allocation constraint stating that one channel can be assigned to more than one SU at a time, and also one SU can gain access to more than one channel at a time. A dedicated Common Control Channel (CCC) is assumed in the network for communicating with the $C_{\mathrm{E}}$. Different structures used in the model are: 
- Channel availability matrix $(C)$ : to represent the spectrum opportunities of all SUs. $C=\left\{c_{i j} \mid c_{i j} \in\{0,1\}\right\}_{N \times M}$, where $c_{i j}=1$ if channel $j$ is sensed as available at $\mathrm{SU} i$, and $c_{i j}=0$ otherwise.

- Interference matrix $(X)$ : to represent interference restrictions among SUs. $X=\left\{x_{i j} \mid x_{i j} \in\{0,1\}\right\}_{N \times N}$, where $x_{i k}=1$ if SUs $i$ and SU $k$ are interfering and cannot be given the same channel simultaneously, and $x_{i k}=0$ otherwise.

- Channel allocation matrix $(A)$ : to represent allocations of the available channels. $A=\left\{a_{i j} \mid a_{i j} \in\{0,1\}\right\}_{N \times M}$, where $a_{i j}=1$ if channel $j$ is allocated to SU $i$, and $a_{i j}=0$ otherwise.

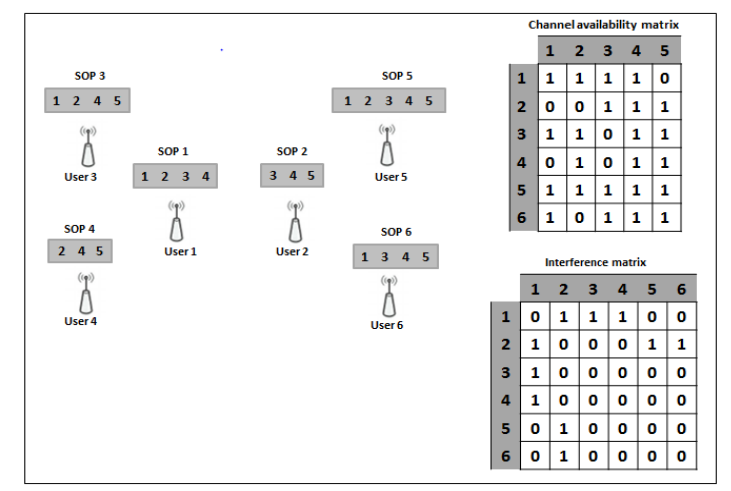

Fig. 1. Diagrammatic representation of the system model

Fig. 1 shows a diagrammatic representation of the system model with six SUs and five available channels along with their channel availability matrix and interference matrix. From the figure we can state that SU 1 cannot be assigned channel 5 since it is unavailable at the SU. And SU 1 and SU 2 must be given different channels to prevent the interference between them. In the similar manner, we can find more such relations between the rest of the SUs and the channels from the Fig. 1. Moreover, the proposed model considers that the channels are heterogeneous with respect to their bandwidth, which implies that different channel may incur a different bandwidth value. The PU of every channel $j$ reserves a certain portion of its total bandwidth which is given as $v_{j}$, where $0<v_{j}<B_{j}\left(B_{j}\right.$ being the bandwidth of channel $j$ ), and as such, the sum total of the requested bandwidth (in the form of reward values) from the SUs who get assigned to channel $j$ should not exceed $B_{j}-v_{j}$. The reserved bandwidth $v_{j}$ can be used as the guard bands when multiple SUs try to access the channel, whereas $B_{j}-v_{j}$ is the bandwidth available for access by the SUs. Therefore, the problem for the proposed mechanism can be defined as follow: Given reward values from SUs for every available channel as according to their SOP. Channel allocation is to be carried out such that the total reward value from the winning SUs of a channel should not exceed the predetermined value of the channel while adhering to the interference and allocation constraints. Accordingly, this approach tries to improve the spectrum utilization so, as to handle the spectrum scarcity problem in today's communicative world. 


\subsection{Spectrum allocation mechanism}

The proposed spectrum allocation mechanism allows a sequential channel allocation with interference and allocation limitations such that SUs get a fair share of the available channels. $C_{\mathrm{E}}$ has knowledge on the channels that are not being used by PUs and their respective predetermined bandwidth values which the PUs want to retain for themselves. SUs are willing to acquire the spectrum for their performance improvement. Initially, every SU $i$ senses for its available channels and maintains them in a SOP list $\Lambda_{i}$. This is because, all the idle channels may not be available to an SU due to different hardware constraints occurring in the sensing phase. Now, if a channel $j \in \Delta$ is one of the channels present in $\Lambda_{i}$ of SU $i$, then channel availability matrix maintained at $C_{\mathrm{E}}$ shows $c_{i j}=1$ implying that $\mathrm{SU} i$ can make a request to access the channel $j$. Thereafter, for allowing spectrum reuse in the proposed model, we apply distance-based criterion to collect the interference status between the SUs while assuming that all SUs transmit at the same power level. And finally, $C_{\mathrm{E}}$ starts the allocation process by taking the channels one after another in a sequence. For allocating a channel $j, C_{\mathrm{E}}$ announces the channel and its predetermined value $v_{j}$. If an $\mathrm{SU} i$ has the channel $j$ in its SOP list, then SU $i$ shows an interest for the channel to $C_{\mathrm{E}}$ by forwarding a reward value, $r_{j i}$, representing the bandwidth that $\mathrm{SU} i$ wants to acquire from channel $j$. So, a reward vector, $R_{j}$, gets formed at $C_{\mathrm{E}}$ such that $R_{j}=\left\{r_{j i}\right\}_{1 \times N}$ which holds the reward values from the SUs for channel $j$. But, if $c_{i j}=0$, then $r_{j i}=0$ since channel $j$ is unavailable at SU $i$. Also, if SU $i$ finds that its bandwidth requirement is higher than $v_{j}$, SU $i$ does not opt to get the channel as to which $r_{j i}=0$. This gives the reward vectors $R_{1}, R_{2}, \ldots, R_{M}$ for $M$ channels. Then, it is assumed that PU retains a small amount of the total channel bandwidth which is given by the value $v_{j}$ for channel $j$. So, if $B_{j}$ is the total bandwidth of channel $j$, then maximum bandwidth available for the use of SUs is $B_{j}-v_{j}$. So, according to the problem definition, the sum total of reward values from the winning SUs for channel $j$ should not exceed the value $B_{j}-v_{j}$. That is,

$$
\sum_{i=1}^{N} a_{i j} r_{j i} \leq B_{j}-v_{j} \quad \forall j \in \Delta .
$$

Also, the channel allocation takes the interference status of SUs into consideration and assigns a channel $j$ to SU $i$ and SU $k$ simultaneously only when they show no interference amongst them along with other design requirements. That is

$$
a_{i j}+a_{k j} \leq 1 \text { if } x_{i k}=1, c_{i j}=1 \text { and } c_{k j}=1 \quad \forall i, k \in \Omega \text { and } \forall j \in \Delta \text {. }
$$

Therefore, considering the above discussions, we design a simple centralized spectrum allocation approach as described in Algorithm 1. According to the algorithm, to allocate a channel $j$, reward vector $R_{j}$ is taken as the input. Then, by taking every SU $i$ one-by-one, we obtain their reward values. If $r_{j i}=0$, we move to the next SU in order. Otherwise, it is checked whether the channel has been assigned to any other SU $k$. If this is true, we get the interference status of the SU $i$ and SU $k$. If $x_{i k}=1, \mathrm{SU} i$ cannot be assigned the channel $j$ and we take the next SU. Otherwise, if $x_{i k}=0$, then we check whether $r_{j k}+r_{j i} \leq B_{j}-v_{j}$. If the condition gets satisfied, 
then we assign channel $j$ to SU $i$. That is, $a_{i j}=1$. If not, then we look for the next SU. Moreover, if the channel $j$ has not been allotted to any other SU earlier, then SU $i$ can directly get the channel without checking any network constraint. Finally, after going through all the SUs, total reward value for the channel is computed from $A$ and $R_{j}$. As such, this process is repeated for every available channel.

With Algorithm 1, idle channels are distributed amongst the SUs while abiding by the network constraints. But it may result in an unfair allocation as because the model considers the SUs that appear earlier in the sequence for channel allocation. This may not proffer a better use of the radio spectrum in all cases. Therefore, with the incentive to build up the spectrum utilization, we extend our work to design a centralized fair spectrum allocation model as discussed in Algorithms 2 and 3. For the extended model, a group matrix, $W=\left\{w_{i k}\right\}_{N \times N}$, is maintained where $w_{i k}=1$ if $\mathrm{SU} i$ and SU $k$ are chosen to be assigned a common channel, otherwise $w_{i k}=0$. And every time a channel is to be allocated, $W$ is initialized to 0 . The centralized fair allocation approach consists of two phases to complete the allocation process. Algorithm 2 designs the first phase of the model, i.e., group formation phase, where different combinations of SUs for the sharing channel are computed while keeping with the allocation and interference constraints and these combinations are maintained in the matrix $W$. Thereafter, Algorithm 3 plans the second phase, i.e., channel allocation phase, where the group of SUs whose total reward value sums up to be the highest among all other groups is obtained from $W$ and the channel gets assigned to the members of that group.

Algorithm 1. Centralized spectrum allocation

Input: $R_{j}$ for allocating channel $j \forall j \in \Delta$

Output: Channel allocation matrix $A$

Step 1. sum $=0 ;$ maxSum $=0$; prevSum $=0 ;$ prevMaxSum $=0 ;$ flag $=0$

Step 2. for $i=1$ to $N$ do

Step 3. prevSum $=$ sum

Step 4. prevMaxSum $=$ maxSum

Step 5. if $r_{j i}=0$ then

Step 6. $\quad a_{i j}=0$

Step 7. else

Step 8. for $m=1$ to $i$ do

Step 9. if $a_{m j}=0$ then

Step 10. flag $=0$

Step 11. else

Step 12.

Step 13.

Step 14.

Step 15.

Step 16.

Step 17.

Step 18.

Step 19.

$$
\text { else }
$$

$$
\text { if } x_{m i}=1 \text { then }
$$

flag $=1$

break

else

$$
\text { flag }=0
$$

end if

Step 20. if flag $=1$ then 


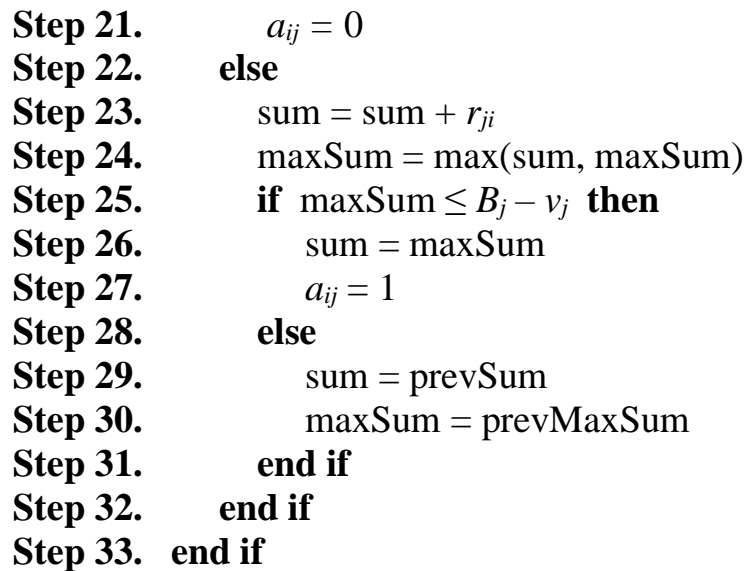

Step 34. end for

We start with the Algorithm 2, where $R_{j}$ is taken as the input to allocate channel $j$. Then, we take the reward values of every SU $i$ one after another. If $r_{j i}=0$, then the $i$-th row of $W$ remains unchanged and we leave away SU $i$ and take up the next SU in sequence. Otherwise, if $r_{j i}=1$, then $w_{i i}$ is set to 1 implying that channel $j$ is given to SU $i$. After assigning the channel to $\mathrm{SU} i$, we look for other SUs who can also share the channel along with SU $i$. We take all other SUs (except SU $i$ ) one after another. On taking an SU $k, k \neq i$, if $r_{j k} \neq 0$, then we check for the value $x_{i k}$. If $x_{i k}=1$, we leave SU $k$ since it is interfering with SU $i$ and move to the next SU. But, when $x_{i k}=0$, we check whether $r_{j k}+r_{j i} \leq B_{j}-v_{j}$. On satisfying the condition, channel $j$ is allotted to SU $k$ along with SU $i$ and $w_{i k}=1$. But, if the condition does not hold, then the next $\mathrm{SU}$ is brought up and this continues till we check the last SU in sequence.

Algorithm 2. Group formation for centralized fair spectrum allocation

Input: $R_{j}$ for allocating channel $j, \forall j \in \Delta$

Output: SU groups obtained in group matrix $W$

Step 1. $W=0$;

Step 2. for $i=1$ to $N$ do

Step 3. sum $=0 ; \operatorname{maxSum}=0 ;$ prevSum $=0 ; \operatorname{prevMaxSum}=0$; flag $=0$;

Step 4. if $r_{j i}=0$ then

Step 5. $\quad w_{i i}=0$;

Step 6. else

Step 7. $\quad w_{i i}=1$;

Step 8. $\quad$ sum $=\operatorname{sum}+r_{j i}$

Step 9. $\max \operatorname{Sum}=\max$ (sum, maxSum);

Step 10. for $k=1$ to $N$ do

Step 11. if $r_{j k}=0$ then

Step 12. $\quad w_{i k}=0$;

Step 13. else

Step 14. $\quad$ prevSum = sum;

Step 15. $\quad$ prevMaxSum = maxSum;

Step 16. if $x_{i k}=0$ then

Step 17. for $m=1$ to $k-1$ do 
Step 18.

Step 19.

Step 20.

Step 21.

Step 22.

Step 23.

Step 24.

Step 25.

Step 26.

Step 27.

Step 28.

Step 29.

Step 30.

Step 31.

Step 32.

Step 33.

Step 34.

Step 35.

Step 36.

Step 37.

Step 38.

Step 39.

Step 40.

Step 41.

Step 42.

Step 43.

Step 44.

Step 45.

Step 46.

Step 47.

Step 48.

Step 49.

Step 50.

Step 51.

Step 52.

Step 53. end i

Step 54. end for

This process is repeated to form different groups of SUs for the channel $j$. Subsequently, Algorithm 3 takes the matrix $W$ as its input and looks to compute the SU group whose reward value for the shared channel sums up to be the highest. As such, the SUs in the winning group get access to the channel while satisfying the design constraints. Looking for the time complexity of Algorithm 2 it can be observed that the outermost loop runs $N$ times. For every $i$-th iteration of the outermost loop, the first inner loop runs $N$ times and for every $k$-th iteration of the first inner loop, the second inner loop runs $k$ times. So, if $m=i$ then

else

if $w_{i m}=0$ then

else

if $x_{m k}=1$ then

flag $=1$;

break;

else

flag $=0$;

end if

end if

end for

else

end if

if flag $=1$ then

$$
w_{i k}=0 \text {; }
$$

elseif flag $=-1$ then

else

$\operatorname{sum}=\operatorname{sum}+r_{j k} ;$

$\operatorname{maxSum}=\max ($ sum, $\operatorname{maxSum})$;

if $\operatorname{maxSum} \leq B_{j}-v_{j}$ then

sum $=\operatorname{maxSum}$;

$w_{i k}=1$;

else

sum $=$ prevSum;

end if

end if

end if

end for 


$$
T(n)=N(1+2+3+\ldots+N)=N\left(\frac{N(N+1)}{2}\right)=\left(\frac{N^{3}+N^{2}}{2}\right)=O\left(N^{3}\right) .
$$

Similarly, for Algorithm 3, the outer loop runs $N$ times and for every $i$-th iteration of outer loop, the inner loop runs $N$ times again, which therefore gives the time complexity of $O\left(N^{2}\right)$ for Algorithm 3.

\subsection{Illustrative example}

In this section, we illustrate an example showing spectrum allocation where we apply both approaches to decide channel allocation. We consider a CR network with 10 SUs and 1 vacant channel. Fig. 2 shows a diagrammatic view of the discussed example. Reward values and interference status of SUs are provided in the matrices $R_{1}$ and $X$ respectively as shown in the figure. Let the bandwidth $B_{1}$ of the channel be $10 \mathrm{~Hz}$ and the predetermined bandwidth value, $v_{1}$ for the channel is set to be $0.2 \mathrm{~Hz}$. So, the available bandwidth is $9.80 \mathrm{~Hz}$. First, the scenario takes up the centralized spectrum allocation scheme to allocate the channels where simply the SUs that appear earlier in the sequence and satisfy the design constraints are considered one-by-one. Therefore, SUs 1, 5 and 8 are taken for allocating the channel such that their reward values sum up to be 5.78. Now, looking to the centralized fair spectrum allocation approach where different SU groups are formed and amongst them, the one that provides highest total reward value is chosen for allocation. Matrix $W$ shows the combinations of SUs that can opt to get the channel. On obtaining the total of reward values for all the combinations, it can be seen that the total value from the SUs 3, 5 and 9 sum to be 9.69 which appears to be the highest among other groups and accordingly the SUs gain access to the shared channel. Hence, the second approach outperforms in providing a better utilization of the radio spectrum in a CR network.

Algorithm 3. Channel allocation for centralized fair spectrum allocation

Input: Group matrix $W$ for allocating channel $j \forall j \in \Delta$

Output: Channel allocation matrix $A$

Step 1. $\max V$ alue $=0$; chosenSU $=\phi$;

Step 2. for $i=1$ to $N$ do

Step 3. value $=0 ; S U=\phi$;

Step 4. for $k=1$ to $N$ do

Step 5. if $w_{i k} \neq 0$ then

Step 6. $\quad$ value $=$ value $+r_{j k}$;

Step 7. $\mathrm{SU}=\mathrm{SU} \cup\{k\}$

Step 8. end if

Step 9. end for

Step 10. if value $>$ maxValue then

Step 11. $\max$ Value $=$ value;

Step 12. $\quad$ chosenSU $=\mathrm{SU}$;

Step 13. end if

Step 14. end for

Step 15. for $i=1$ to $N$ do

Step 16. if $i \in$ chosenSU then 
Step 17. $\quad a_{i j}=1$;

Step 18. end if

Step 19. end for

\section{Performance evaluation}

This section provides an overview of the performance evaluation being carried out through MATLAB based simulations to validate the proposed model. We deploy a CR network where SUs are randomly distributed in an area of size $600 \mathrm{~m} \times 600 \mathrm{~m}$. Interference between SUs is modelled by considering the physical distance between the SUs. For performance analysis, we first evaluate the proposed centralized fair allocation mechanism by comparing its results for different sets of SUs when numbers of channels are also varied. Secondly, we compare both the proposed algorithms and an existing channel allocation mechanism called PreDA [22] for different performance metrics. PreDA is an auction based mechanism where the CR constraint for dynamics in spectrum opportunities has been included during the allocation process. To allow multi-winner and multi-channel allocation, PreDA uses a group formation algorithm which returns groups of non-interfering SUs. Then for each channel, virtual grouping is carried out (from every group) so that virtual groups of SU who are interested to get that particular channel are considered when the channel is to be allocated. To resolve compatibility issue with our model, we have applied the grouping and virtual grouping methods of PreDA to get the noninterfering groups of SUs. Then for a channel, we take the virtual groups formed for that channel and get the virtual group with the highest group value. If the group value does not exceed the limit set for the channel, then the SUs in the virtual group are assigned the channel. Otherwise, we move to the group having next highest group value. This is continued until we get a virtual group, which gets the channel, or we cover all the virtual groups but could not allocate the channel. So, a channel remains unassigned if there appears no virtual group for the channel which satisfies the predefined bandwidth condition explained in Section 3.2. To perform the simulation study, two different network scenarios are considered. In one scenario, number of SUs is varied from 10 to 60 keeping the number of channels fixed at 6 , and in the other scenario, number of channels is varied from 4 to 14 keeping the number of SUs fixed at 40. For both of these scenarios, all results of the simulation are averaged over 500 rounds. Third, we carry out a random allocation to evaluate the performance of spectrum utilization and spectrum reuse in a CR network deployed with 20 SUs and 6 free channels. To perform the random experiment we pick SUs for each channel using the simple random sampling method. The SUs are picked constrained to the condition that the total bandwidth requirement of the chosen SUs does not exceed the bandwidth limit of the channel. This is repeated for all the channels in a trial. We take the mean as the population parameter and get the mean spectrum utilization and mean spectrum reuse values from the samples collected in the trials. The results of random allocation are compared with the proposed centralized fair allocation model by varying the number of trails from 100 to 1000 . 

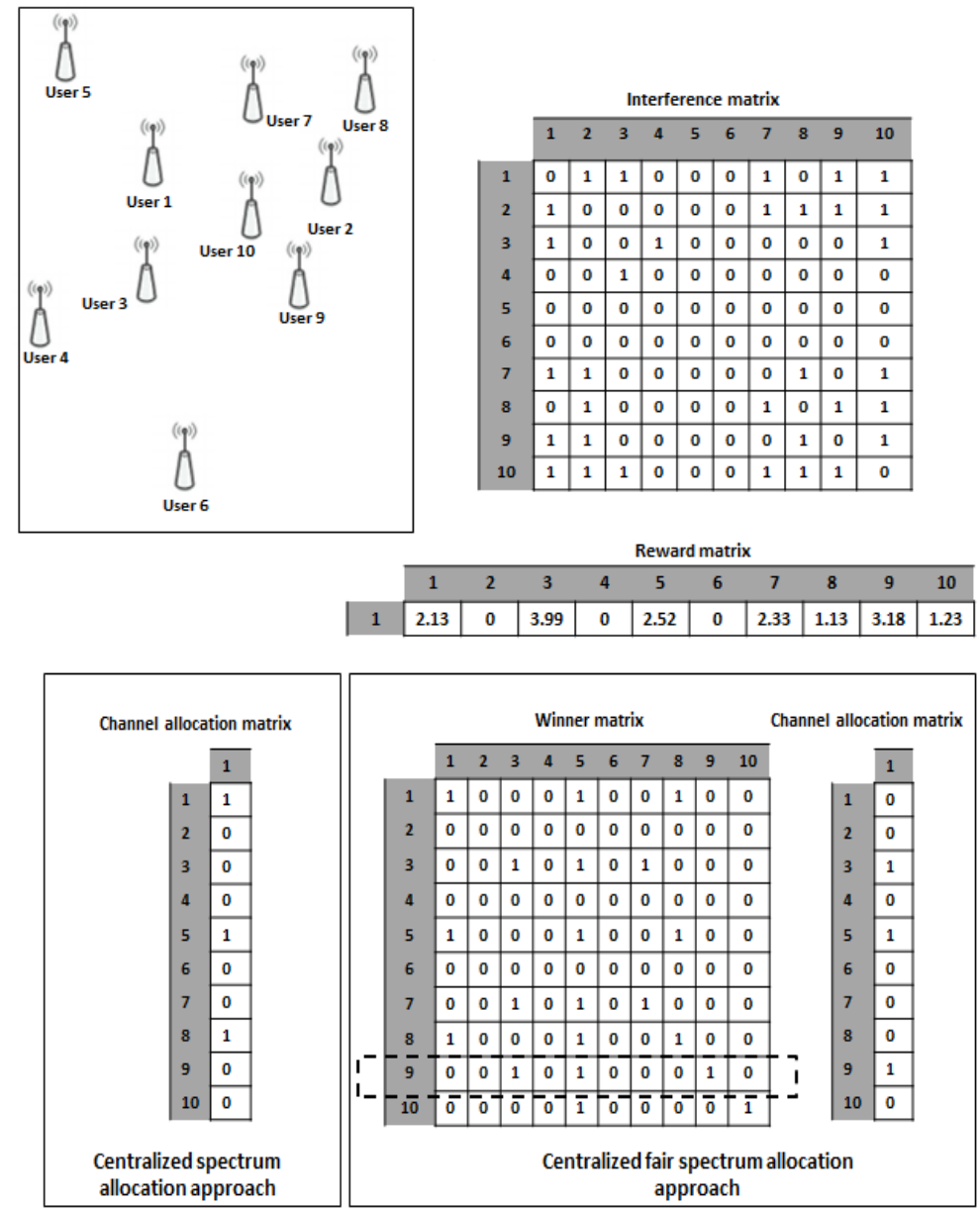

Fig. 2. Example illustrating the proposed spectrum allocation mechanism

The performance metrics considered for evaluation are as follows.

- Spectrum utilization $\left(S_{\mathrm{u}}\right)$ : This is the sum of winning reward values for every allocated channel.

$$
S_{\mathrm{u}}=\sum_{j=1}^{M} \sum_{i=1}^{N} a_{i j} r_{j i} .
$$

- User satisfaction $\left(U_{\mathrm{s}}\right)$ : This is a ratio of number of winning SUs to the total number of participating SUs in the network.

$$
U_{\mathrm{s}}=\frac{N-\sum_{i=1}^{N} \prod_{j=1}^{M}\left(1-a_{i j}\right)}{N} .
$$

- Spectrum reuse $\left(S_{\mathrm{r}}\right)$ : This is a ratio of winning SUs to the number of channels, which have been assigned during the allocation mechanism. 


$$
S_{\mathrm{r}}=\frac{\sum_{j=1}^{M} \sum_{i=1}^{N} a_{i j}}{M-\sum_{j=1}^{M} \prod_{i=1}^{N}\left(1-a_{i j}\right)} .
$$

- Throughput $\left(T_{\mathrm{r}}\right)$ : Throughput for the network earned by the allocation $A$ is given as follows.

$$
T_{\mathrm{r}}=r_{j i} \log _{2}\left(1+P_{\mathrm{t}} \frac{P_{\mathrm{L}(i)}}{I_{(i)}+\sigma^{2}}\right) .
$$

This model assumes that all channels have the same maximum allowable transmission power, $P_{\mathrm{t}}=0.01 . \sigma^{2}$ is the noise variance and taken as $10^{-5}$ for all channels, $P_{\mathrm{L}(i)}$ is the path loss factor between $i$-th SU's transmitter and receiver, and $I_{\mathrm{i}}$ is the interference from PUs; $r_{j i}$ is the reward value of winner SU $i$ for channel $j$.

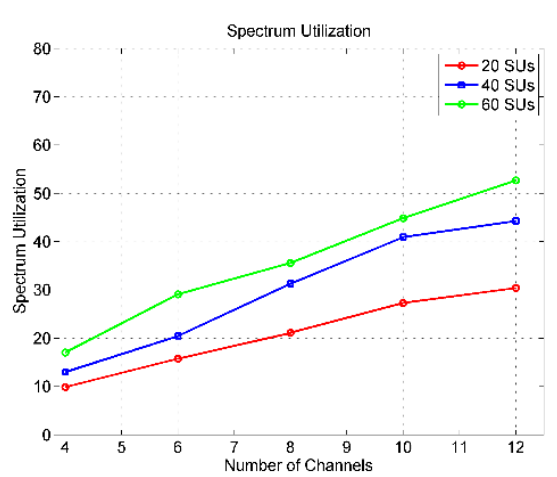

a

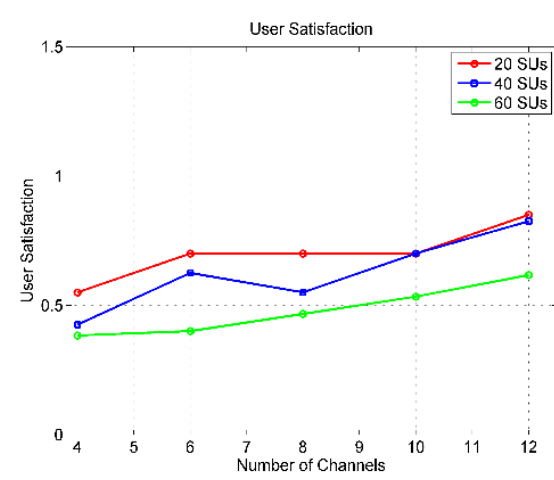

$\mathrm{b}$

Fig. 3. Spectrum utilization and user satisfaction respectively for different sets of SUs with varying number of channels

Fig. 3a shows the spectrum utilization on implementing the centralized fair allocation approach when number of channels is varied for different sets of SUs.

From the figure it can be observed that on increasing the number of channels, spectrum utilization shows a growth for all sets of SUs. This occurs because with increase in number of channels, more spectra become available among the users and they get more chance to acquire the spectrum. Also, spectrum utilization moderately increases when the SU set has more members for a given number of channels. Increasing number of SUs provide a wider range of reward values as to which total reward values for a channel may rise relatively thus resulting in a growth in spectrum usage. Similarly, in Fig. 3b, user satisfaction improves with increase in the number of channels for every SU set since higher availability of the spectrum resource allows more number of SUs to benefit from them. But, for a given number of channels, user satisfaction degrades slowly with increasing number of SUs as because there will be greater competition for the limited available resource. 


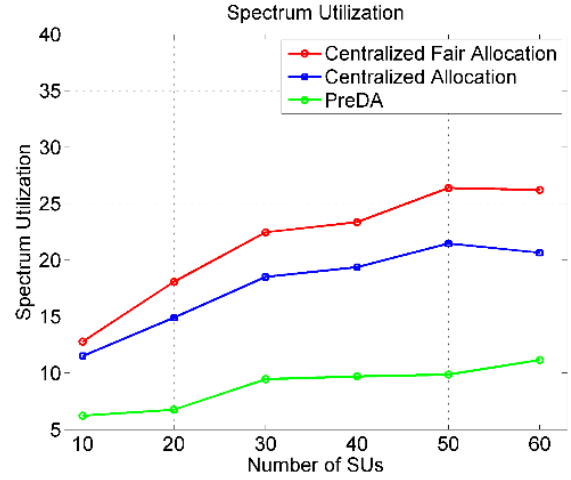

a

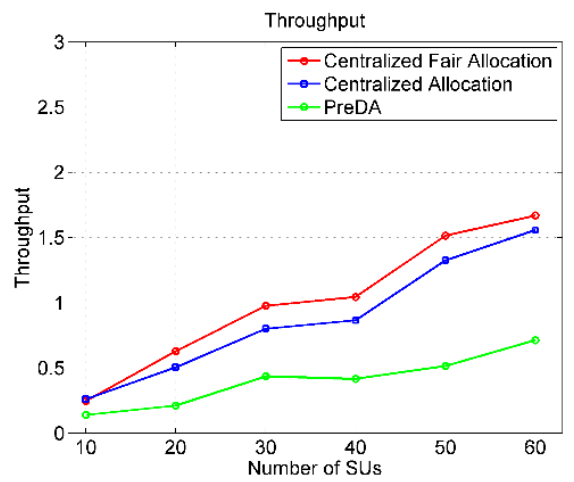

c

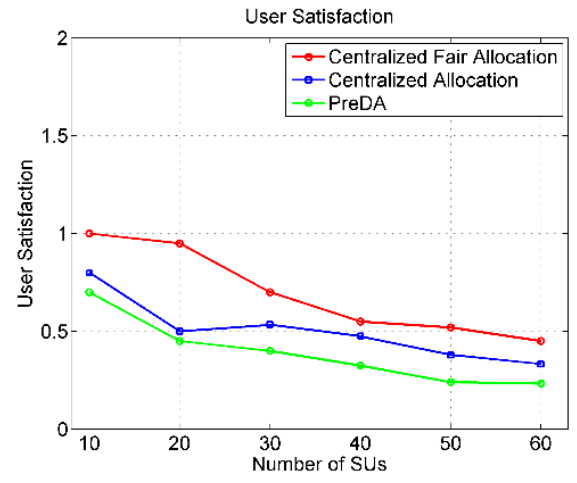

b

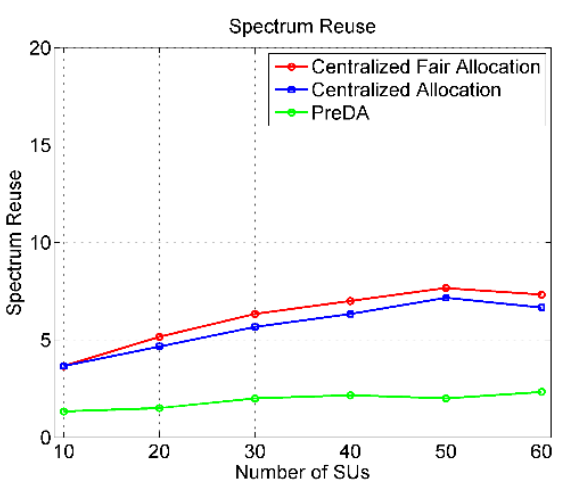

d

Fig. 4. Spectrum utilization, user satisfaction, throughput and spectrum reuse respectively with respect to number of SUs

In Fig. 4a a comparison for spectrum utilization is shown among the proposed schemes, i.e., centralized spectrum allocation and centralized fair spectrum allocation, along with PreDA. With increase in number of SUs, spectrum utilization increases because more number of SUs are participating to use the free channels. Also, number of non-interfering SUs who can reuse a channel increases on increasing the SU count. Spectrum utilization for the fair allocation model gives a better performance as compared to the other two approaches. This is due to the allocation strategy applied in the model where all combinations of SUs that opt to get the channel are considered and the SU group whose total reward value sums up to be the highest is chosen for channel allocation. On considering the simple centralized approach, channel allocation does not look for all SU combinations due to which we may not achieve a good allocation pattern under certain situations. When number of SUs is less, both of the proposed approaches show nearly similar results for spectrum utilization because the range of reward values for the channels is not much large. In PreDA, a channel is allocated to one of the virtual groups formed for the channel. But such a group of SUs does not provide a good use of the radio resource because of which PreDA shows a degraded performance. Fig. $4 \mathrm{~b}$ shows the user satisfaction for the two proposed models and PreDA. Centralized fair allocation approach gives a preferably good performance because the count of winning SUs is more in this 
approach. On increasing the number of SUs, user satisfaction shows a decreasing trend because for same number of channels more SUs are competing to get their desired spectrum. The number of winning SUs can also differ due to interference and channel availability conditions in the network. In few cases, user satisfaction in PreDA may show a moderate growth in the result than the simple centralized approach. This is because there appears some channel in PreDA that is allocated to a group having more number of SUs but it is not an SU group with a high reward value. Fig. 4c shows that the throughput attained by the centralized fair allocation outperforms the other schemes with changing number of SUs. This is due to the virtual grouping method applied in PreDA, which takes lesser number of SUs in the groups formed for a channel. Spectrum reuse, as shown in Fig 4d, increases with increasing number of SUs. With less number of SUs, both proposed approaches may show similar results. But when number of SU increases, the wide range of reward values from each SU needs to be checked for allocating a channel. This gives a better performance as we observe in the centralized fair allocation model.

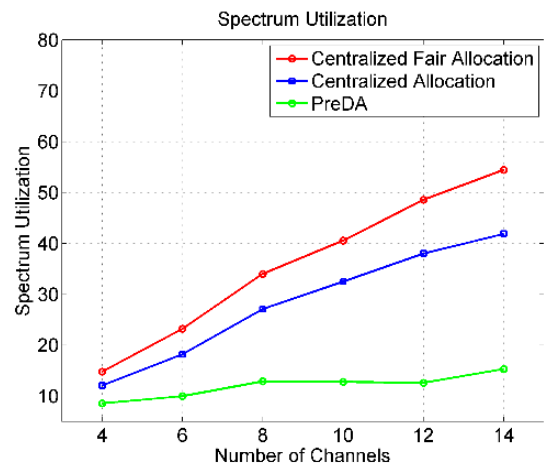

a

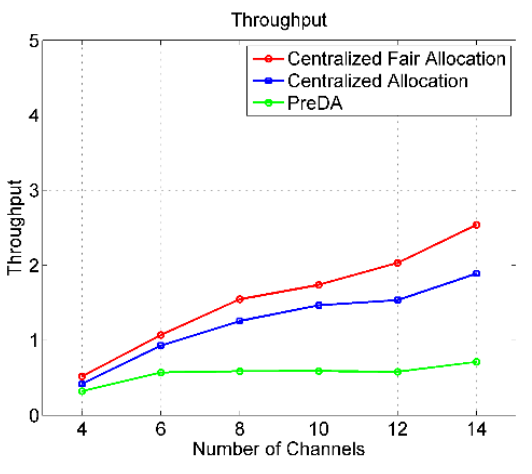

$\mathrm{c}$

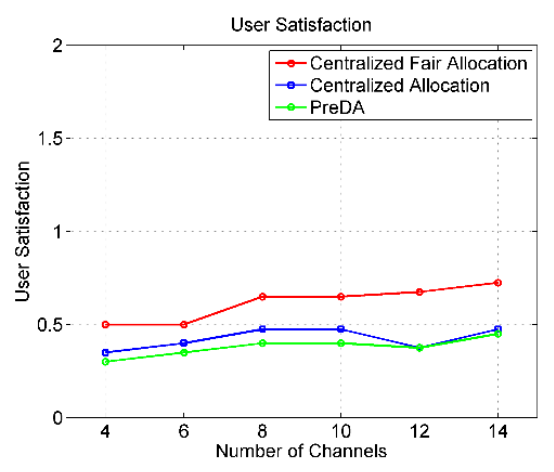

b

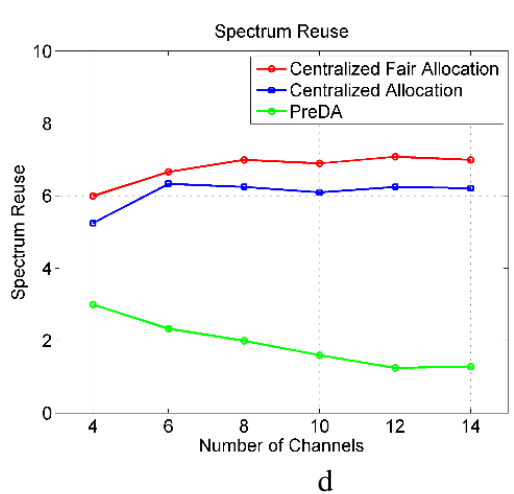

Fig. 5. Spectrum utilization, user satisfaction, throughput and spectrum reuse respectively with respect to number of channels

In Fig. 5a, the proposed allocation approaches are compared with PreDA to analyse the spectrum utilization with changing number of channels. From the figure, it can be observed that the centralized fair allocation model provides a far better utilization of the spectrum bands than the simple centralized model and PreDA. Moreover for all the models, spectrum utilization increases with increase in number 
of channels because with more number of channels more spectra become available amongst the SU set. This allows greater number of SUs to obtain the free channels.

The spectrum utilization is PreDA remains low due to virtual grouping of SUs to allocate the channels. Fig. 5b shows the user satisfaction obtained in the two proposed models and PreDA. When channels are more in number, there appears more number of winning SUs in the SU set due to which user satisfaction increases. The fair allocation method gives good result as compared to the other two methods. However, user satisfaction for simple centralized allocation and PreDA show moderate variations because the allocation strategy applied in both these models does not consider the SU group with high reward values. Fig. 5c gives the throughput values for all the models where we can observe that the fair allocation model performs better due to its designed allocation mechanism. Also, Fig. 5d shows that the fair allocation model gives a much improved performance for spectrum reuse. Deteriorated spectrum reuse in PreDA accounts to the virtual groups formed in PreDA, which assigns lesser number of SUs to a channel.

Fig. $6 \mathrm{a}$ and $6 \mathrm{~b}$ compares our proposed fair allocation method with a random allocation where the simulation is carried out for different sets of trials. The results obtained from the two figures infer that for each set of trial the proposed model gives a far better performance in terms of spectrum utilization as well as spectrum reuse. Hence, from the simulation results it can be concluded the centralized fair allocation approach significantly enhances the network performance.

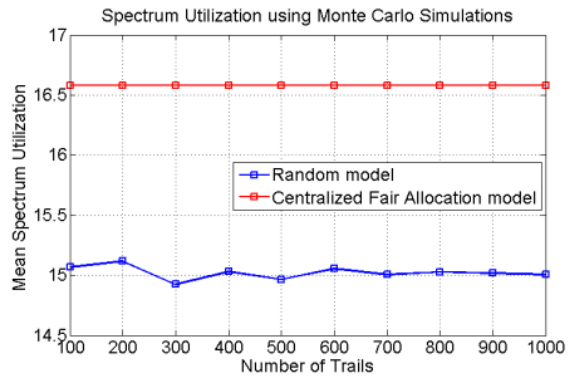

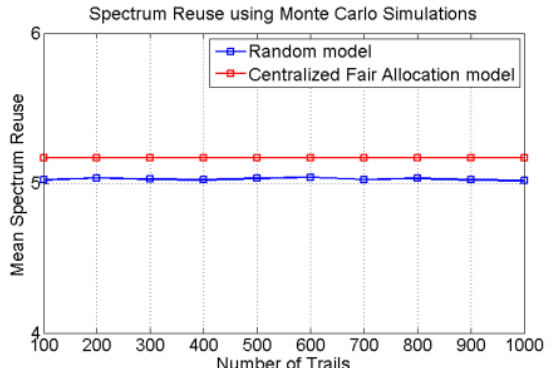

b

Fig. 6. Spectrum utilization and spectrum reuse respectively with respect to number of trials carried out in simulation

\section{Conclusion}

In this paper we have developed a spectrum allocation mechanism for CRN which can distribute the free channels amongst SUs such that utilization of the radio spectrum can be significantly improved. Available channels are shared sequentially while allowing both multi-channel and multi-winner allocation. Interference amongst SUs restricts such users from using a common channel simultaneously. Moreover, spectrum opportunities of the SUs show a dynamic behaviour in CRN and this has been taken care of during the allocation process in this model. Finally, two allocation approaches have been designed which accommodate all the network constraints so that the network throughput does not get degraded. Simulations carried out for 
assessing the network performance reveal that the centralized fair spectrum allocation model outperforms other approaches in terms of spectrum utilization and provides a better network throughput in CRN. As a future research direction, we can plan to design a distributed spectrum allocation approach for CRN which deploys all the design constrains considered in this model.

Acknowlegement: This paper is an extended version of a paper presented in International Conference on Advanced Computational and Communications Paradigm (ICACCP 2017), Sikkim, India.

\section{References}

1. FCC. FCC Document ET Docket No Spectrum Policy Task Force Report. - Federal Communications Commission, 2002, pp. 02-135.

2. A kyildiz, I. F., W.-Y. Lee, M. C. Vuran, S. Moh ant y. Next Generation/Dynamic Spectrum Access/Cognitive Radio Wireless Networks: A Survey. - Elsevier Computer Networks, Vol. 50, 2006, pp. 2127-2159.

3. X ing, X., T. J ing, W. Ch en g, Y. H u o, X. Ch e n g. Spectrum Prediction in Cognitive Radio Networks. - IEEE Wireless Communications, Vol. 20, 2013, pp. 90-96.

4. $\mathrm{Hu}$, F., B. Ch e n, K. Z h u. Full Spectrum Sharing in Cognitive Radio Networks Toward 5G: A Survey. - IEEE Access, Vol. 6, 2018, pp. 15754-15776.

5. S o n g, M., C. X i n, Y. Z h a o, X. C h e n g. Dynamic Spectrum Access: from Cognitive Radio to Network Radio. - IEEE Wireless Communications, Vol. 19, 2012, No 1, pp. 23-29.

6. Trag o s, E. Z., S. Z e a d a 11 y, A. G. Fragki a d a k i s, V. A. S i r i s. Spectrum Assignment in Cognitive Radio Networks: A Comprehensive Survey. - IEEE Communications Surveys \& Tutorials, Vol. 15, 2013, No 3, pp. 1108-1135.

7. Xiang, J., Y. Zhang, T. Skeie. Medium Access Control Protocols in Cognitive Radio Networks. - Wireless Communications and Mobile Computing, 2009, pp. 1-18.

8. D e vi, M., N. S a r m a, S. K. D e k a. Spectrum Allocation in Cognitive Radio Networks - A Centralized Approach. - In: Proc. of International Conference on Advanced Computational and Communications Paradigm (ICACCP'17), 2017, pp. 92-100.

9. A h m e d, E., A. G a n i, S. A b o $1 \mathrm{f} \mathrm{a} \mathrm{z} 1$ i, L. J. Y a o, S. U. K h a n. Channel Assignment Algorithms in Cognitive Radio Networks: Taxonomy, Open Issues, and Challenges. - IEEE Communications Surveys \& Tutorials, Vol. 18, 2016, pp. 795-823.

10. Ni y ato, D., E. Hos s a in. A Game-Theoretic Approach to Competitive Spectrum Sharing in Cognitive Radio Networks. - In: Proc. of IEEE International Conference on Wireless Communications and Networking (WCNC'07), 2007, pp. 16-20.

11. Z h e n g, H., C. P e n g. Collaboration and Fairness in Opportunistic Spectrum Access. - In: Proc. of IEEE International Conference on Communications (ICC'05), 2005, pp. 3132-3136.

12. Khaledi, M., A. A. Abouzeid. Auction-Based Spectrum Sharing in Cognitive Radio Networks with Heterogeneous Channels. - Information Theory and Applications Workshop (ITA'13), 2013.

13. Zhao, Z., Z. Peng, S. Zheng, J. Shang. Cognitive Radio Spectrum Allocation Using Evolutionary Algorithms. - IEEE Transactions on Wireless Communications, Vol. 8, 2009, pp. 4421-4425.

14. Z h a n g, W., L. D e n g, Y. C. K i a t. Dynamic Spectrum Allocation for Heterogeneous Cognitive Radio Network. - In: Proc. of IEEE International Conference on Wireless Communications and Networking (WCNC'16), 2016.

15. D a r s e n a, D., G. G e 11 i, F. V e r d e. An Opportunistic Spectrum Access Scheme for Multicarrier Cognitive Sensor Networks. - IEEE Sensors Journal, Vol. 17, 2017, pp. 2596-2606.

16. Li, C., W. Li u, Q. Li u, C, Li. Spectrum Aggregation Based Spectrum Allocation for Cognitive Radio Networks. - In: Proc. of IEEE International Conference on Wireless Communications and Networking (WCNC'14), 2014, pp. 1626-1631. 
17. W a n g, X., Z. Li, P. X u, Y. X u, X. G a o, H.-H. Ch e n. Spectrum Sharing in Cognitive Radio Networks-An Auction Based Approach. - IEEE Transactions on Systems, Man and Cybernetics, Vol. 40, 2010, pp. 587-596.

18. Ka s h, I. A., R. Murth y, D. C. Parke s. Enabling Spectrum Sharing in Secondary Market Auctions. - IEEE Transactions on Mobile Computing, Vol. 13, 2014, pp. 556-568.

19. H y d e r, C. S., T. D. J e it s c h k o, L. X i a o. Bid and Time Truthful Online Auctions in Dynamic Spectrum Markets. - IEEE Transactions on Cognitive Communications and Networking, Vol. 3, 2017, pp. 82-96

20. S h i, Z., G. L u o. Multi-Band Spectrum Allocation Algorithm Based on First-Price Sealed Auction. - Cybernetics and Information Technologies, Vol. 17, 2017, No 1, pp. 104-112.

21. Zh a i, X., T. Zh o u, C. Zhu, B. Ch en, W. F an g, K. Z h u.Truthful Double Auction for Joint Internet of Energy and Profit Optimization in Cognitive Radio Networks. - IEEE Access, 2018, pp. 23180-23190

22. K h a i r u 11 a h, E. F., M. Ch a t t e r j e e. PreDA: Preference-Based Double Auction for Spectrum Allocation in Heterogeneous DSA Networks. - Computer Communications, Vol. 133, 2019, pp. 41-50.

23. D on g, W., S. Ra 11 a pa 11 i, L. Qi u, K. K. R a makrishn a n, Y. Zh a n g. Double Auctions for Dynamic Spectrum Allocation. - IEEE/ACM Transactions on Networking, Vol. 24, 2016, pp. 2485-2497.

24. Z h a n g, X., D. Y a n g, G. X u e, R. Y u, J. T a n g. Transmitting and Sharing: A Truthful Double Auction for Cognitive Radio Networks. - In: Proc. of IEEE International Conference on Communications (ICC'18), 2018.

Received: 01.08.2020; Second Version: 26.02.2021; Third Version: 23.03; Accepted: 12.04.2021 